\title{
Synthesis of Highly Stable Cobalt Nanomaterial Using Gallic Acid and Its Application in Catalysis
}

\author{
Saba Naz, ${ }^{1,2,3}$ Abdul Rauf Khaskheli, ${ }^{4,5}$ Abdalaziz Aljabour, ${ }^{5}$ Huseyin Kara, ${ }^{2,6}$ \\ Farah Naz Talpur, ${ }^{3}$ Syed Tufail Hussain Sherazi, ${ }^{2,3,6}$ Abid Ali Khaskheli, ${ }^{3}$ and Sana Jawaid ${ }^{3}$ \\ ${ }^{1}$ Dr. M. A. Kazi Institute of Chemistry, University of Sindh, Jamshoro 76080, Pakistan \\ ${ }^{2}$ Department of Chemistry, Faculty of Science, Selcuk University, 42075 Konya, Turkey \\ ${ }^{3}$ National Centre of Excellence in Analytical Chemistry, University of Sindh, Jamshoro, Pakistan \\ ${ }^{4}$ Department of Pharmacy, Shaheed Mohtarma Benazir Bhutto Medical University, Larkana 77150, Pakistan \\ ${ }^{5}$ Advanced Technology Research and Application Center, Selcuk University, 42075 Konya, Turkey \\ ${ }^{6}$ Department of Biotechnology, Faculty of Science, Necmettin Erbakan University, 42090 Konya, Turkey
}

Correspondence should be addressed to Saba Naz; saba0208@gmail.com

Received 10 February 2014; Accepted 20 May 2014; Published 11 August 2014

Academic Editor: Young-Seok Shon

Copyright (C) 2014 Saba Naz et al. This is an open access article distributed under the Creative Commons Attribution License, which permits unrestricted use, distribution, and reproduction in any medium, provided the original work is properly cited.

\begin{abstract}
We report the room temperature $\left(25-30^{\circ} \mathrm{C}\right)$ green synthesis of cobalt nanomaterial $(\mathrm{CoNM})$ in an aqueous medium using gallic acid as a reducing and stabilizing agent. $\mathrm{pH} 9.5$ was found to favour the formation of well dispersed flower shaped CoNM. The optimization of various parameters in preparation of nanoscale was studied. The AFM, SEM, EDX, and XRD characterization studies provide detailed information about synthesized CoNM which were of $4-9 \mathrm{~nm}$ in dimensions. The highly stable CoNM were used to study their catalytic activity for removal of azo dyes by selecting methyl orange as a model compound. The results revealed that $0.4 \mathrm{mg}$ of CoNM has shown $100 \%$ removal of dye from $50 \mu \mathrm{M}$ aqueous solution of methyl orange. The synthesized CoNM can be easily recovered and recycled several times without decrease in their efficiency.
\end{abstract}

\section{Introduction}

Metal nanoparticles have attracted much attention in nanoscale science and engineering technology over the past decades due to their unusual chemical and physical properties, such as catalytic activity, novel electronic, and opti$\mathrm{cal}$ and magnetic properties. Their main application areas include catalysts, absorbents, chemical and biological sensors, optoelectronics, information storage, and photonic and electronic devices [1]. Cobalt nanomaterial (CoNM) exhibits high resistance to oxidation, corrosion, and wear. CoNM have been prepared by several synthetic methods including solvothermal process [2], thermal decomposition method [3], hydrothermal microemulsion process [4], high temperature solution phase method [5], and reduction by $\mathrm{NaBH}_{4}$ at room temperature [6]. Among all, the wet chemical reduction method has the advantage over the others in easy control of the reaction process. However, most of the wet chemical reduction methods reported to date rely strongly on the use of environmentally and biologically hazardous organic solvents and reducing agents (i.e., hydrazine, sodium borohydride, dimethyl formamide, formaldehyde, sodium hypophosphite, or hydroxylamine hydrochloride, etc.) [1].

Recently, there is an increased emphasis on the subject of green chemistry, to avoid the problems related to toxic chemicals and solvents. Nanomaterials prepared by green rout are environmentally benevolent. Raveendran et al. [7] prepared silver nanoparticles using water as a solvent, $\beta$-D-glucose as a reducing agent, and starch as a protecting agent. Liu et al. [8] synthesized gold nanocrystals using $\beta$-D-glucose as both the reducing and stabilizing agent. In addition Xiong et al. [1], worked on the synthesis of highly stable nanosized copper particles with an average particle size of less than $2 \mathrm{~nm}$ using a nontoxic L-ascorbic acid as a reducing and capping agent; 
precursor in aqueous medium was studied by Xiong et al. [1]. Moreover Martinez-Castanon et al. [9] worked on the synthesis and antibacterial activity of silver nanoparticles with gallic acid in an aqueous chemical reduction method. Gallic acid is a natural poly-phenolic compound that can be used as a reducing agent [10]. To our best knowledge, the nanomaterials with urchinlike and flowerlike architectures can be used in catalysis because of their high specific surface area [11].

Here, we tried firstly one-step method for the synthesis of biocompatible highly stable flower shape CoNM, by using natural gallic acid as reductant and stabilizing agent at the room temperature without any additional protecting reagents. We also monitor the catalytic activity of synthesized CoNM by selecting methyl orange (MO) azo dyes for their removal by adsorption. Regarding the toxic effect of dyes, studies have been carried out that show the extensive release of a number of azo dyes (such as MO) from effluents of the textile industry and many other sources which cause aquatic environmental pollution leading to severe health problems in aquatic life [12]. Numerous approaches have been introduced to purify the waters including biodegradation, ion exchange, and adsorption [13]. However, these processes are insufficient to control the pollution because they basically perform transformation of the hazards from one phase to another and need additional costs for treatments like incineration or land filling to terminate the end product [14]. In view of the risky effects of the dye, we have fabricated small size CoNM via greener route that possess marvelous potential as catalytic materials to completely degrade $\mathrm{MO}$ azo dye, which can be taken as a model for other degradable dyes.

Because of the magnetism of CoNM, they can be recovered by a magnet after adsorption. Hence, it can be applied to use in the field of catalysis and wastewater treatment, which may play important roles in the future of industrial effluents. The effect of gallic acid moles to cobalt metal on the size and shape of CoNM as well as their characteristic was also investigated. UV-Vis and IR spectroscopy, scanning electron microscopy (SEM), and X-ray diffraction (XRD) were employed in the characterization of the prepared CoNM [10].

\section{Experimental}

2.1. Chemicals and Reagents. $\mathrm{CoCl}_{2} \cdot 6 \mathrm{H}_{2} \mathrm{O}$ (99.9\%), gallic acid (99\%), MO dye (99\%), and sodium hydroxide (98\%) were purchased from Sigma-Aldrich, ACS reagent. All chemicals were of analytical grade and were used as received without further purification. Ultrapure water was used for preparation of CoNM.

2.2. Preparation of Stock Solutions. Stock solutions of $0.1 \mathrm{M}$ $\mathrm{CoCl}_{2} \cdot 6 \mathrm{H}_{2} \mathrm{O}$ and $0.1 \mathrm{M}$ gallic acid were prepared in $100 \mathrm{~mL}$ volumetric flasks using the required quantities of each and diluted to the mark with ultrapure CoNM in aqueous medium. Further, $1 \mathrm{M} \mathrm{NaOH}$ solution was prepared to maintain desired pH. 0.001 M stock solution of MO dye was prepared in ultrapure water to carry out the catalytic activity of CoMN for reduction/degradation of dye.

2.3. Procedure for Fabrication of CoNM. The synthesis of CoNM was carried out at room temperature by using gallic acid as reductant and stabilizer. Typically, a $10 \mathrm{~mL}$ aqueous solution containing $1 \times 10^{-3} \mathrm{M} \mathrm{CoCl}_{2} \cdot 6 \mathrm{H}_{2} \mathrm{O}$ solution was taken. To this solution $1.0 \mathrm{~mL}$ of $1 \times 10^{-3} \mathrm{M}$ solution of gallic acid is added under continuous stirring and then the resulting solution was adjusted to $\mathrm{pH}$ value 9.5 by addition of $1 \mathrm{M} \mathrm{NaOH}$ solution. The reaction solution was left for $10-$ $15 \mathrm{~min}$ to confirm the completion of reduction reaction. The solution color was observed to change from light pink to blue and then brown after adjusting $\mathrm{pH}$ 9.5. After $10 \mathrm{~min}$, no further change in color took place, indicating that the reactions were complete. As-prepared gallic acid derived CoNM obtained in an aqueous medium were separated by magnet or simple decantation of remaining aqueous phase. The collected CoNM were then dried for 24 hours in air. The solution was analyzed by UV-Vis spectroscopy and it was found that the as-prepared sample of CoNM was stable up to 60 days.

2.4. Instrumentation. The UV-Vis spectroscopic absorbance was taken using UV Probe 2.35 spectrophotometer (shimadzu). Fourier transform infrared (FTIR) spectra of standard gallic acid and CoNM were recorded using a Vertex 70 (Bruker, Germany) with Platinum ATR Diamond. Atomic force microscopy (AFM) images were recorded using a NTMDT, NTEGRA (Russia) AFM, MFM, and Nanoscope IV controller. Scanning electron microscopy (SEM) EVO LS 10, AEISS (England), images were taken on aluminum sample holder. Energy dispersive X-ray analysis EDX was completed with Bruker $123 \mathrm{eV}$ (Germany). The X-ray diffraction (XRD) pattern of synthesized nanoparticles was recorded using a Bruker Advance D8 XRD instrument, equipped with $\mathrm{Cu} \mathrm{K} \alpha$ source (wavelength $=1.5406)$. The XRD pattern was obtained in powder mode.

2.5. Sample Preparation for AFM Studies. In a typical AFM analysis, $10-20 \mu \mathrm{L}$ volumes of dispersed solution of CoNM were put through drop casting method on glass cover slip and heated at $60^{\circ} \mathrm{C}$ for $30 \mathrm{~min}$ followed by air drying up to $5 \mathrm{~min}$ to ensure binding of CoNM with the glass surface and thereby loss of water molecules.

2.6. Sample Preparation for SEM/EDX Studies. Small quantities of dispersed solution of CoNM in aqueous medium were mounted on aluminum sample holder (Gold sputter quarter $7 \mathrm{~nm}$ ), by a dip coating method and forwarded for vacuum drying in a Cressington Sputter Coater, Auto 108, in the presence of Argon gas for 2 min to clean the surface and ensure that solvent had been removed. The sample prepared in this way was also used for EDX studies.

2.7. Sample Preparation for XRD and FTIR Studies. Solid state CoNM capped with gallic acid was dried and poured into 
a glass tube. As-prepared Co nanoparticles were utilized for $\mathrm{XRD}$ and FTIR analysis without any additional pretreatment.

2.8. Catalytic Test for Reduction of Dye. The catalytic activity of CoNM was inspected for reduction/degradation of MO dye by putting $0.4 \mathrm{mg}$ of CoNM in a solution having $50 \mu \mathrm{M}$ concentrations of dye and diagnosed with the help of UVVis spectroscopy. Several experiments like dosage of catalyst, concentration of dye solution, and time study were performed to find out the catalytic performance of CoNM.

\section{Results and Discussions}

3.1. Co Nanomaterial Synthesized with Gallic Acid. In this work we present a simple green aqueous method for the fabrication of small size CoNM using gallic acid as reducing and stabilizing agent in the absence of any other capping reagents. The phenol group in the gallic acid molecule is responsible for the reduction of metal ions by providing electron through redox reaction. Gallic acid has two $\mathrm{pKa}$ values, first is 4.1 for the carboxylic group and second is 8.38 for the hydroxyl group [15], and that is why the stabilization of CoNM at higher $\mathrm{pH}$ value could come through the complex formation between oxygen of hydroxyl group and the CoNM. Basically, $\mathrm{pH}$ shows a great role on the size of nanoparticles; therefore, at high $\mathrm{pH}$ value, it could be possible that the complexation of $\mathrm{Co}$ (II) ions by $\mathrm{NaOH}$ decreases its reactivity and slows down the rate of nucleation and growth resulting in a smaller particle size [16]. UV-Vis absorbance spectroscopy is a very useful technique for studying metal nanoparticles because the shapes and positions of peak are sensitive to size of particle. The influence of gallic acid concentration on the UV-Vis absorbance of as-prepared CoNM is shown in Figure 1. As we can see that the surface plasmon band of CoNM has shown a continuous blue shift with the variation in the concentration of gallic acid which indicates the presence of very small size Co nanoparticles. Regarding the above results, the high amount of gallic acid leads to the formation of small size nanoparticles due to its enhanced capping ability.

3.2. XRD Analysis. Figure 2(a) shows the diffraction pattern obtained for the 4-9 nm CoNM; this analysis was made to confirm the identity of the products. The diffractogram shows broad prominent peaks around $2 \theta=47.01^{\circ}$ corresponding to the (111) plane of fcc (face centered cube) cobalt which have a good match with the standard diffraction pattern (JSPDC no. 05-0727) [17]. The broad XRD peak indicates a nanocrystalline nature. Owing to the noisy XRD pattern the other weaker peaks that correspond to the fcc cobalt nanomaterial was not visibly seen in a diffraction pattern [18]. The average crystallite size is determined through X-ray diffraction line broadening by the Debye-Scherrer formula. Consider the following:

$$
D=\frac{K \lambda}{\beta \cos \theta} .
$$

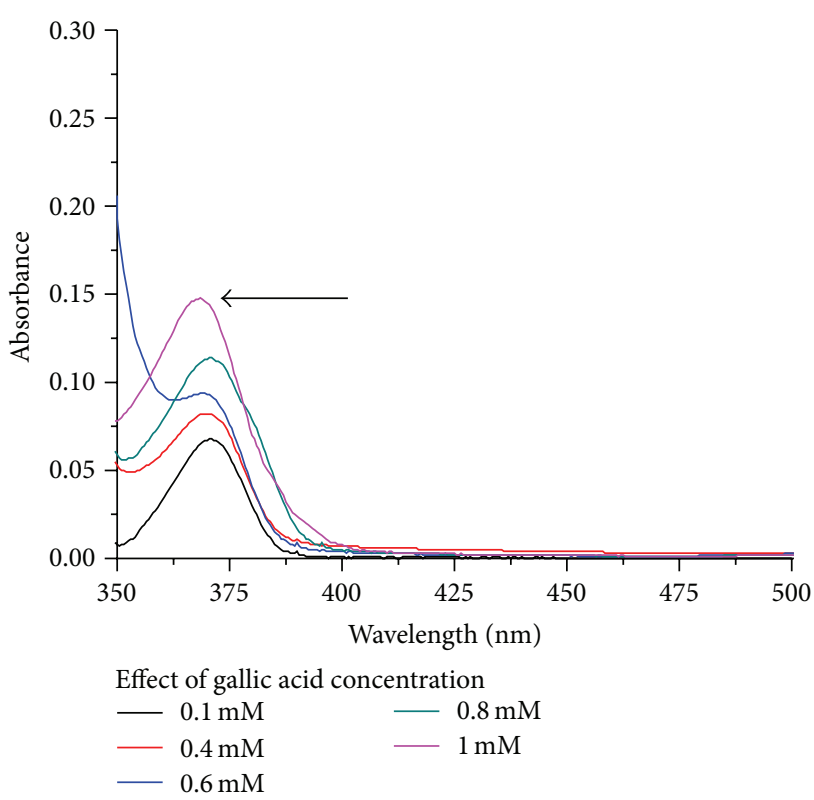

FIGURE 1: The absorption spectra of CoNM synthesized by various amounts of gallic acid to metal at room temperature.

In (1), $D$ shows the average crystallite size, $K=0.89$ is the Scherrer constant, $\lambda=1.5406 \mathrm{~A}^{\circ}$ is the wavelength of $\mathrm{X}$ ray ( $\mathrm{Cu} \mathrm{K} \alpha_{1}$ radiation), $\theta$ is the diffraction angle of the peak, and $\beta$ represents the full width at half maximum of the peaks [19]. The average crystallite size of CoNM was found to be 4$9 \mathrm{~nm}$ which is also confirmed by SAXS pattern as shown in Figure 2(b).

3.3. Fourier Transform Infrared (FTIR) Spectroscopy. IR spectrum was also measured for the further investigation to identify the possible molecular response for efficient stabilization of CoNM. The dried CoNM were used for IR analysis. Figures 3(a) and 3(b) show the infrared spectra of gallic acid standard and CoNM obtained by gallic acid reduction of $\mathrm{CoCl}_{2} \cdot 6 \mathrm{H}_{2} \mathrm{O}$. In Figure $3(\mathrm{a})$, the strong and broad band between 3600 and $2500 \mathrm{~cm}^{-1}$ and the strong and narrow peak at $1702 \mathrm{~cm}^{-1}$ could be assigned to the stretching vibration of $\mathrm{OH}$ group and carbonyl group, which indicated the existence of carboxyl group in the gallic acid. Three peaks observed at 1616, 1541, and $1450 \mathrm{~cm}^{-1}$ are typical stretching vibrations of $\mathrm{C}-\mathrm{C}$ bonds in an aromatic ring. There are several peaks in $1300-1000 \mathrm{~cm}^{-1}$ region that could be assigned to the stretching vibration of $\mathrm{C}-\mathrm{O}$ bond and bending vibration of $\mathrm{O}-\mathrm{H}$ bond of gallic acid. In Figure 3(b), the strong and broad band in the $3650-2700 \mathrm{~cm}^{-1}$ region was considered to be stretching vibration of $\mathrm{OH}$ group, which basically covered $\mathrm{C}-\mathrm{H}$ bond stretching vibration at about $3100 \mathrm{~cm}^{-1}$. Stretching vibration of $\mathrm{C}-\mathrm{O}$ bond and bending vibration of $\mathrm{O}-\mathrm{H}$ bond in $1300-1000 \mathrm{~cm}^{-1}$ region was still retained, but the intensity obviously decreased. In contrast with that of gallic acid shown in Figure 3(a), the IR absorption spectrum of CoNM observed from Figure 3(b) indicated that the stretching vibration peak of carbonyl group shifted from 


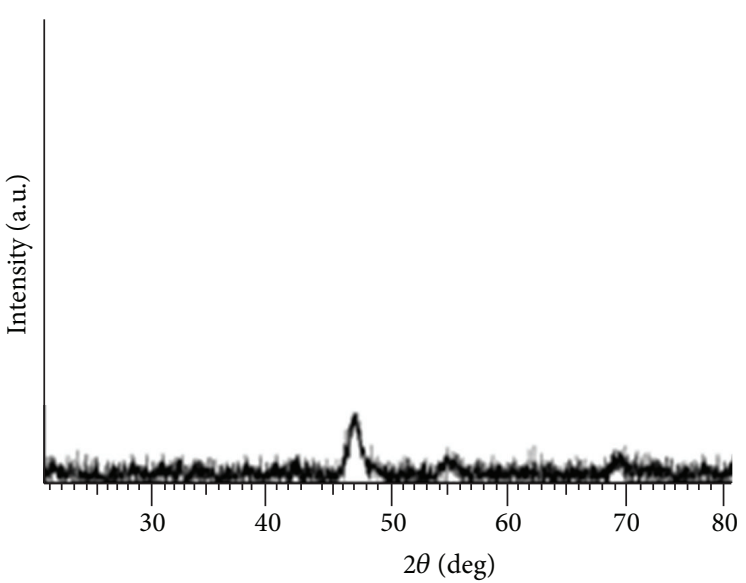

(a)

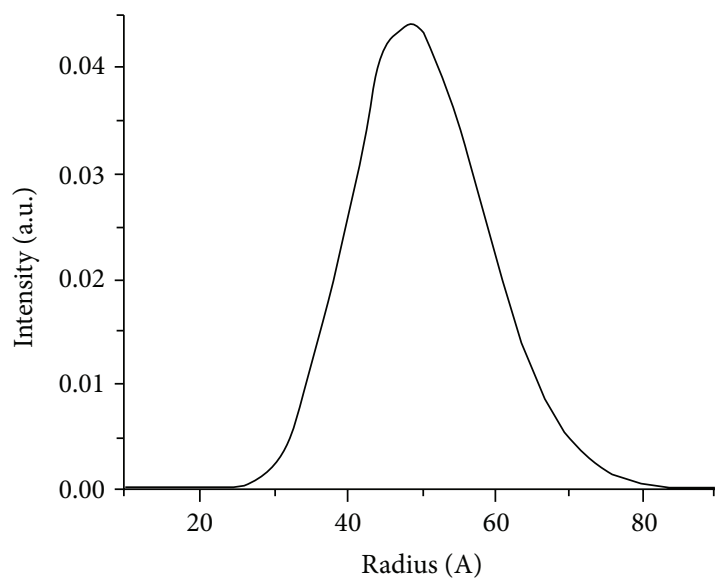

(b)

FIgURE 2: (a) The XRD diffraction pattern for the 4-9 nm CoNM (b) with SAXS profile.

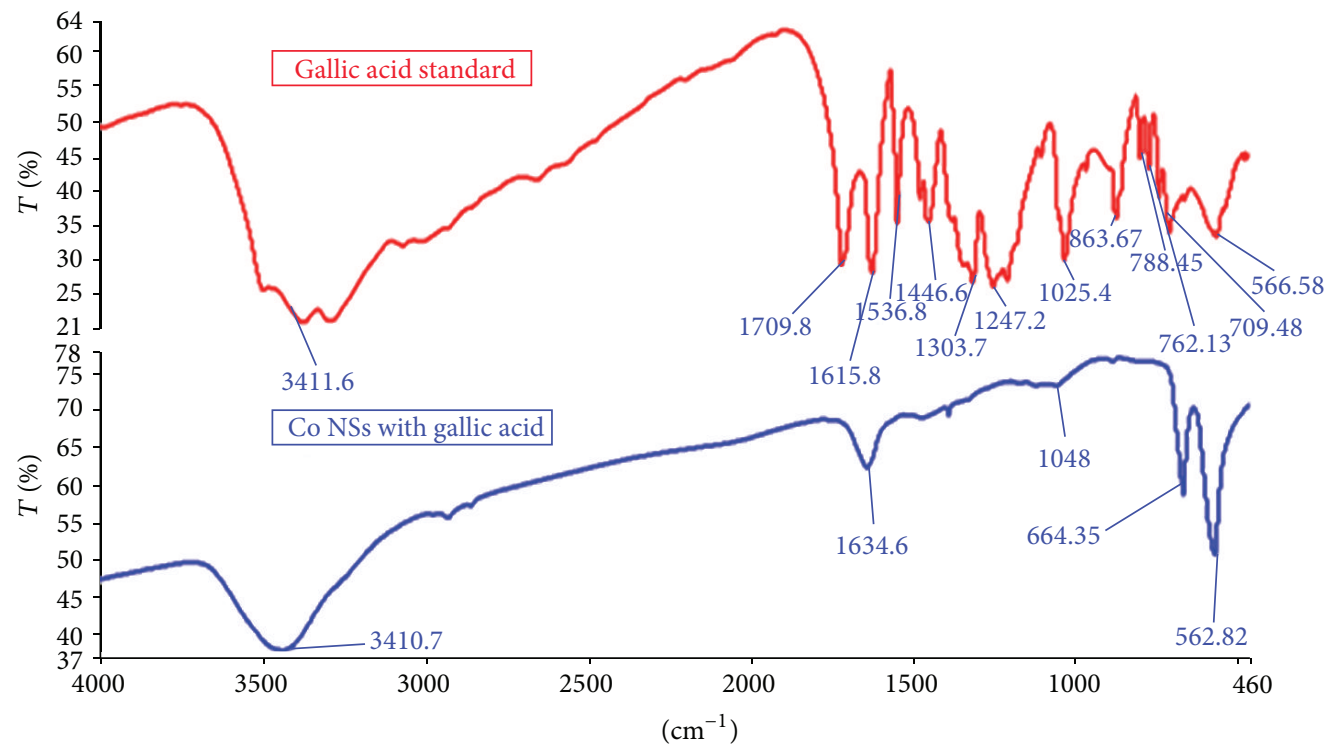

FIGURE 3: (a) FTIR spectra for gallic acid standard and (b) CoNM capped with gallic acid molecule.

1709 to $1634 \mathrm{~cm}^{-1}$, and the stretching vibration of $\mathrm{C}-\mathrm{C}$ bond at 1616 and $1537 \mathrm{~cm}^{-1}$ was covered with a broad and middle intensity band at around $1634 \mathrm{~cm}^{-1}[10]$. Since phenolic compounds are easily oxidized to form quinones, it was speculated that the product of gallic acid reduction of $\mathrm{CoCl}_{2} \cdot 6 \mathrm{H}_{2} \mathrm{O}$ might be a quinoid compound. The results indicated that quinoid compound with keto-enol system might be produced by gallic acid reduction of $\mathrm{CoCl}_{2} \cdot 6 \mathrm{H}_{2} \mathrm{O}$ and absorbed on the surface of CoNM. Usually, when molecule absorbs on the nano scale metal island, surface-enhanced Raman and infrared spectra can be observed [20].

3.4. Morphology of CoNM by SEM and AFM. Scanning electron microscopy was used to investigate the surface morphology, structure, and particle size of CoNM. Figures 4(a) and 4(b) show the SEM images of the synthesized CoNM with two different magnifications. It clearly reveals that uniform flower-like microspheres can be synthesized successfully by a simple reduction of gallic acid. Atomic force micrographs (AFM) present highly distributed CoNM grown after capping with gallic acid molecules on glass cover slips as shown in Figure 4(c). One of the most beneficial features of atomic force microscopy is its ability to quantitatively measure the spectral dimensions of different surface features and high resolution images. The flower images obtained from SEM were relatively similar to AFM images which confirm the formation of nanoflowers shape CoNM.

3.5. Stability Study of CoNM. Stability of synthesized CoNM was assessed for 60 days of storage at ambient room temperature $25 \pm 2^{\circ} \mathrm{C}$ by UV-Vis analysis. There was no change 


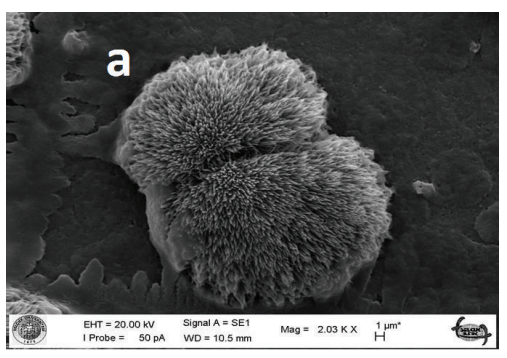

(a)

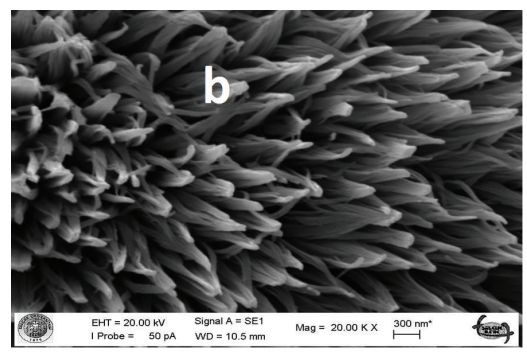

(b)

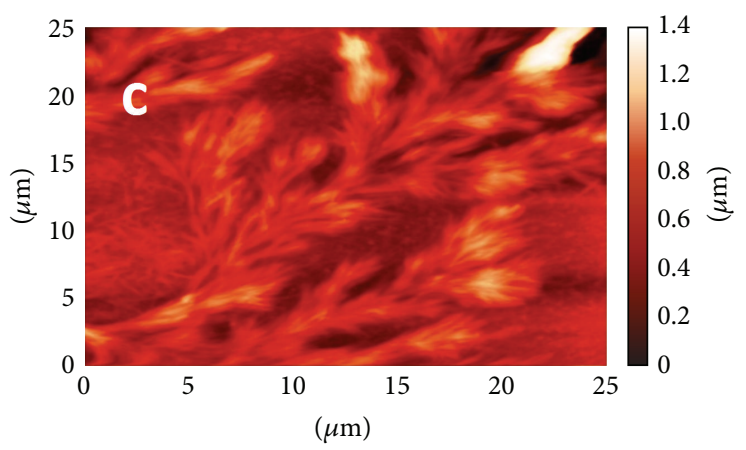

(c)

FIGURE 4: (a) SEM micrographs of CoNM at low magnification, (b) at high magnification, and (c) AFM image of CoNM.

observed in the appearance or color intensity during the entire period of storage time.

3.6. Catalytic Performance of Methyl Orange (MO) Dye. Methyl orange $\left(\mathrm{C}_{14} \mathrm{H}_{14} \mathrm{~N}_{3} \mathrm{NaO}_{3} \mathrm{~S}, \mathrm{MW}=327.33 \mathrm{Da}\right)$ is an intensely colored compound used in dyeing and printing textiles. It is made from sodium nitrite, dimethylaniline, and sulfanilic acid through diazotization process. It is considered to be a harmful and carcinogenic pollutant causing various diseases and disorders in living organisms [6]. UVVis spectroscopy was used to measure the concentration of MO dye in an aqueous solution. The color of MO solution changes from orange to colorless after the addition of CoNM within 50 seconds. Figure 5 shows the decrease of MO dye absorbance with respect to time which suggests the $100 \%$ removal of MO dye within $50 \mathrm{sec}$. The as-prepared CoNM showed high adsorption capacity and a faster reaction rate and thus can be potentially used to remove harmful dyes from aqueous solution within a small time and easily separated from aqueous solution.

3.7. Recovery of CoNM Catalyst after Reuse. The newly synthesized CoNM can be used several times without loss of their catalytic efficiency. In addition the CoNM can be easily recovered after the removal of MO dye then washed with sufficient amount of deionized water followed by drying. Then, the dried CoNM is kept at room temperature. The high catalytic efficiency of CoNM was observed during recycling experiments the results shows that $0.4 \mathrm{mg}$ CoNM can be even eight times reuse for removal of $50 \mu \mathrm{M}$ solution of MO dye within 50 sec each time.

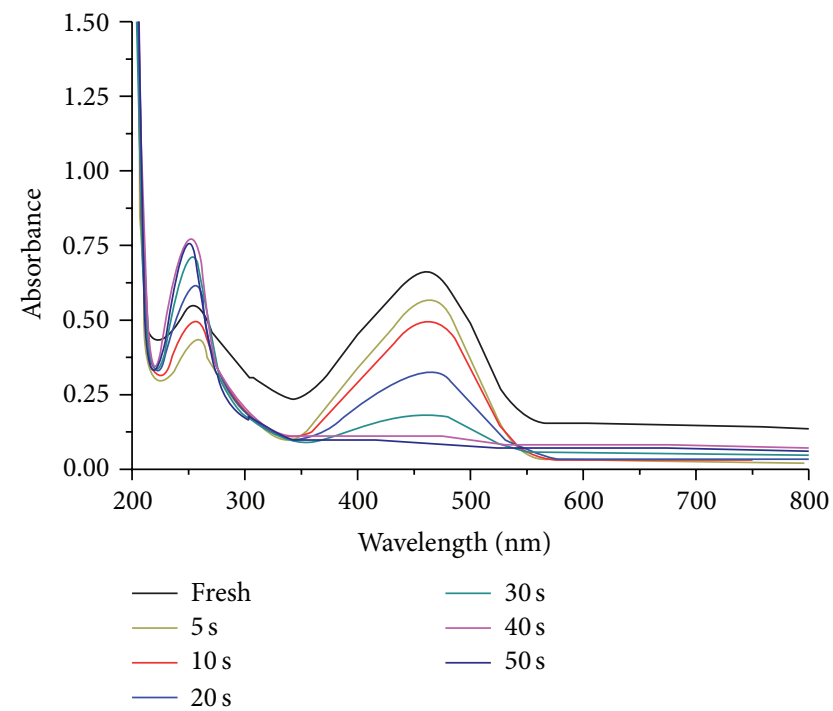

FIGURE 5: UV-Visible spectra of methyl orange in the presence of CoNM.

\section{Conclusions}

In conclusion, we have demonstrated a facile green method to synthesize low cost CoNM 4-9 nm in size on average with a narrow size distribution and a uniform shape by employing gallic acid as both the reducing and capping agent. The prepared dispersions of CoNM are highly stable and do not show any sign of sedimentation even after storage for 60 days. Since the reagents used in the reaction medium are 
completely nontoxic and environmentally friendly, this green method can be readily used for biomedical applications. Moreover, the highly stable freshly prepared CoNM shows highly catalytic performance for MO azo dyes removal within $50 \mathrm{sec}$ at room temperature. The CoNM found to be recovered easily and recycle several times without loss of their catalytic activity.

\section{Conflict of Interests}

The authors declare that they do not have any conflict of interests.

\section{Acknowledgment}

The authors would like to thank TUBITAK (The Scientific and Technological Research Council of Turkey) for the finance support (Program no. 2216).

\section{References}

[1] J. Xiong, Y. Wang, Q. Xue, and X. Wu, "Synthesis of highly stable dispersions of nanosized copper particles using l-ascorbic acid," Green Chemistry, vol. 13, no. 4, pp. 900-904, 2011.

[2] L. P. Zhu, W. D. Zhang, H. M. Xiao, Y. Yang, and S. Y. Fu, "Facile synthesis of metallic Co hierarchical nanostructured microspheres by a simple solvothermal process," The Journal of Physical Chemistry C, vol. 112, no. 27, pp. 10073-10078, 2008.

[3] N. Matoussevitch, A. Gorschinski, W. Habicht et al., "Surface modification of metallic Co nanoparticles," Journal of Magnetism and Magnetic Materials, vol. 311, no. 1, pp. 92-96, 2007.

[4] W. Liu, W. Zhong, X. Wu, N. Tang, and Y. Du, "Hydrothermal microemulsion synthesis of cobalt nanorods and self-assembly into square-shaped nanostructures," Journal of Crystal Growth, vol. 284, no. 3-4, pp. 446-452, 2005.

[5] Y. Su, X. OuYang, and J. Tang, "Spectra study and size control of cobalt nanoparticles passivated with oleic acid and triphenylphosphine," Applied Surface Science, vol. 256, no. 8, pp. 2353-2356, 2010.

[6] X. Liang and L. Zhao, "Room-temperature synthesis of airstable cobalt nanoparticles and their highly efficient adsorption ability for Congo red," RSC Advances, vol. 2, no. 13, pp. 54855487, 2012.

[7] P. Raveendran, J. Fu, and S. L. Wallen, "Completely "green" synthesis and stabilization of metal nanoparticles," Journal of the American Chemical Society, vol. 125, no. 46, pp.13940-13941, 2003.

[8] J. Liu, G. Qin, P. Raveendran, and Y. Ikushima, "Facile "green" synthesis, characterization, and catalytic function of $\beta$-Dglucose-stabilized Au nanocrystals," Chemistry, vol. 12, no. 8, pp. 2131-2138, 2006.

[9] G. A. Martinez-Castanon, N. Nino-Martinez, F. MartinezGutierrez, J. R. Martinez-Mendoza, and F. Ruiz, "Synthesis and antibacterial activity of silver nanoparticles with different sizes," Journal of Nanoparticle Research, vol. 10, no. 8, pp. 1343-1348, 2008.

[10] W. Wang, Q. Chen, C. Jiang, D. Yang, X. Liu, and S. Xu, "Onestep synthesis of biocompatible gold nanoparticles using gallic acid in the presence of poly-( $N$-vinyl-2-pyrrolidone)," Colloids and Surfaces A: Physicochemical and Engineering Aspects, vol. 301, no. 1-3, pp. 73-79, 2007.
[11] Y. Chen, L. Hu, M. Wang, Y. Min, and Y. Zhang, "Self-assembled $\mathrm{Co}_{3} \mathrm{O}_{4}$ porous nanostructures and their photocatalytic activity," Colloids and Surfaces A: Physicochemical and Engineering Aspects, vol. 336, no. 1-3, pp. 64-68, 2009.

[12] K. Zhang and W. C. Oh, "The photocatalytic decomposition of different organic dyes under UV irradiation with and without $\mathrm{H}_{2} \mathrm{O}_{2}$ on $\mathrm{Fe}-\mathrm{ACF} / \mathrm{TiO}_{2}$ photocatalysts," Journal of the Korean Ceramic Society, vol. 46, no. 6, pp. 561-567, 2009.

[13] C. Umpuch and S. Sakaew, "Removal of methyl orange from synthetic wastewater onto chitosan-coated-montmorillonite clay in fixed-beds," GMSARN International Journal, vol. 6, pp. 175-180, 2012.

[14] D. Kamel, A. Sihem, C. Halima, and S. Tahar, "Decolourization process of an azoïque dye (Congo red) by photochemical methods in homogeneous medium," Desalination, vol. 247, no. 1-3, pp. 412-422, 2009.

[15] A. E. Fazary, M. Taha, and Y. H. Ju, "Iron complexation studies of gallic acid," Journal of Chemical \& Engineering Data, vol. 54, no. 1, pp. 35-42, 2008.

[16] K. W. Huang, C. J. Yu, and W. L. Tseng, "Sensitivity enhancement in the colorimetric detection of lead(II) ion using gallic acid-capped gold nanoparticles: improving size distribution and minimizing interparticle repulsion," Biosensors and Bioelectronics, vol. 25, no. 5, pp. 984-989, 2010.

[17] V. V. Matveev, D. A. Baranov, G. Y. Yurkov, N. G. Akatiev, I. P. Dotsenko, and S. P. Gubin, "Cobalt nanoparticles with preferential hcp structure: a confirmation by X-ray diffraction and NMR," Chemical Physics Letters, vol. 422, no. 4-6, pp. 402405, 2006.

[18] N. S. Gajbhiye, S. Sharma, A. K. Nigam, and R. S. Ningthoujam, "Tuning of single to multi-domain behavior for monodispersed ferromagnetic cobalt nanoparticles," Chemical Physics Letters, vol. 466, no. 4-6, pp. 181-185, 2008.

[19] M. Alagiri, C. Muthamizhchelvan, and S. Hamid, "Synthesis of superparamagnetic cobalt nanoparticles through solvothermal process," Journal of Materials Science: Materials in Electronics, vol. 24, no. 11, pp. 4157-4160, 2013.

[20] Q. Song, X. Ai, D. Wang et al., "Preparation of gold/triblock copolymer composite nanoparticles," Journal of Nanoparticle Research, vol. 2, no. 4, pp. 381-385, 2000. 

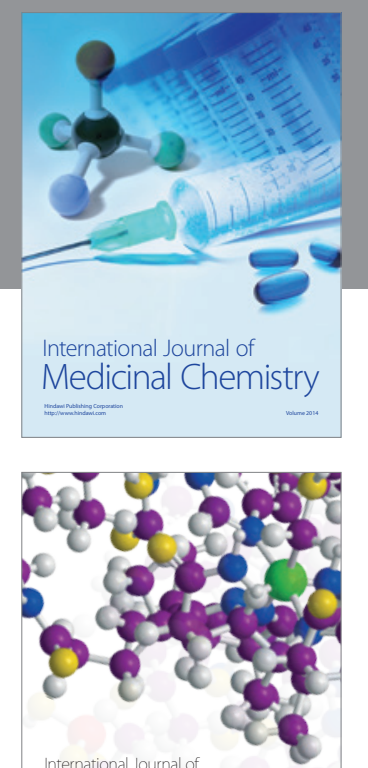

\section{Carbohydrate} Chemistry

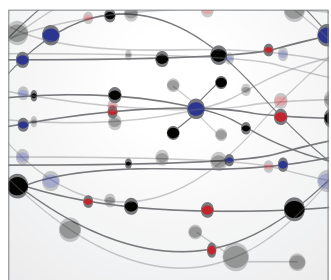

The Scientific World Journal
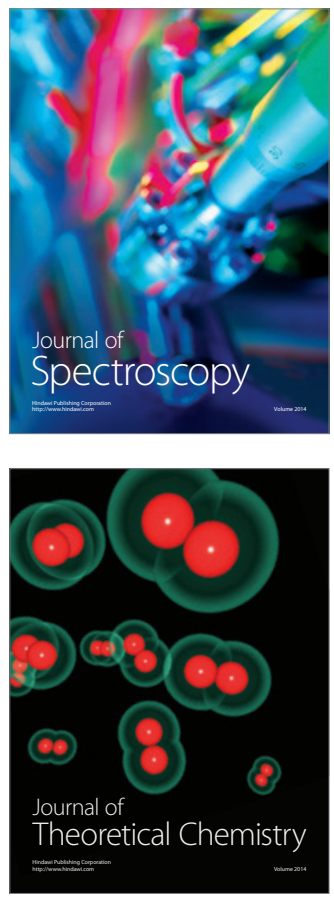
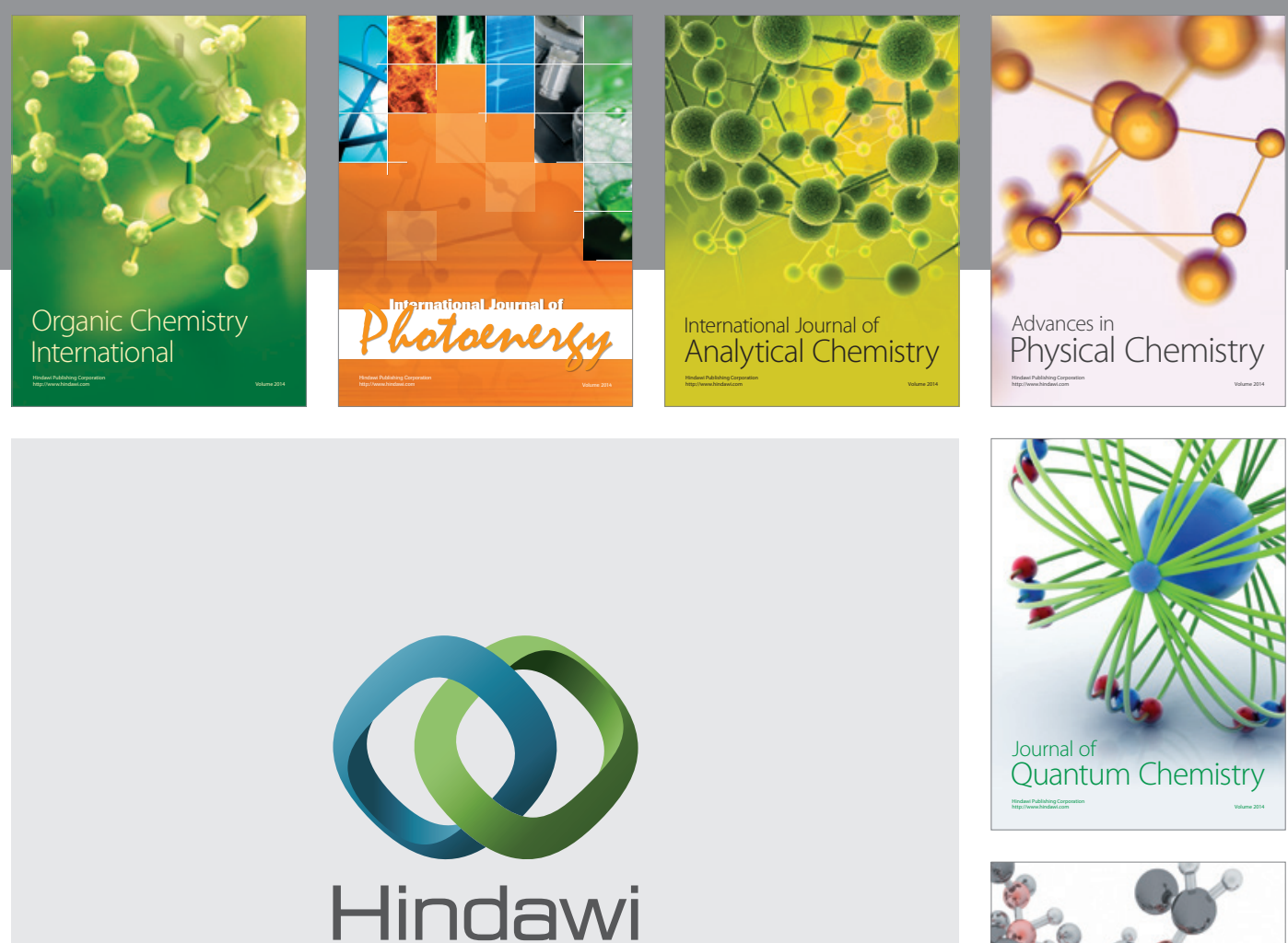

Submit your manuscripts at

http://www.hindawi.com

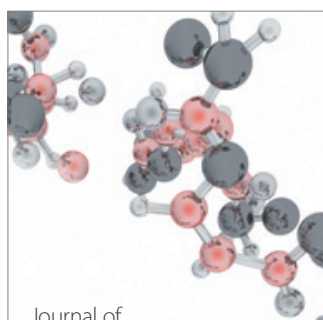

Analytical Methods

in Chemistry

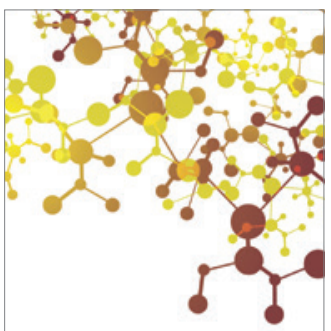

Journal of

Applied Chemistry

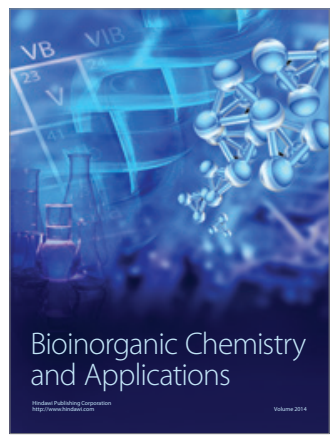

Inorganic Chemistry
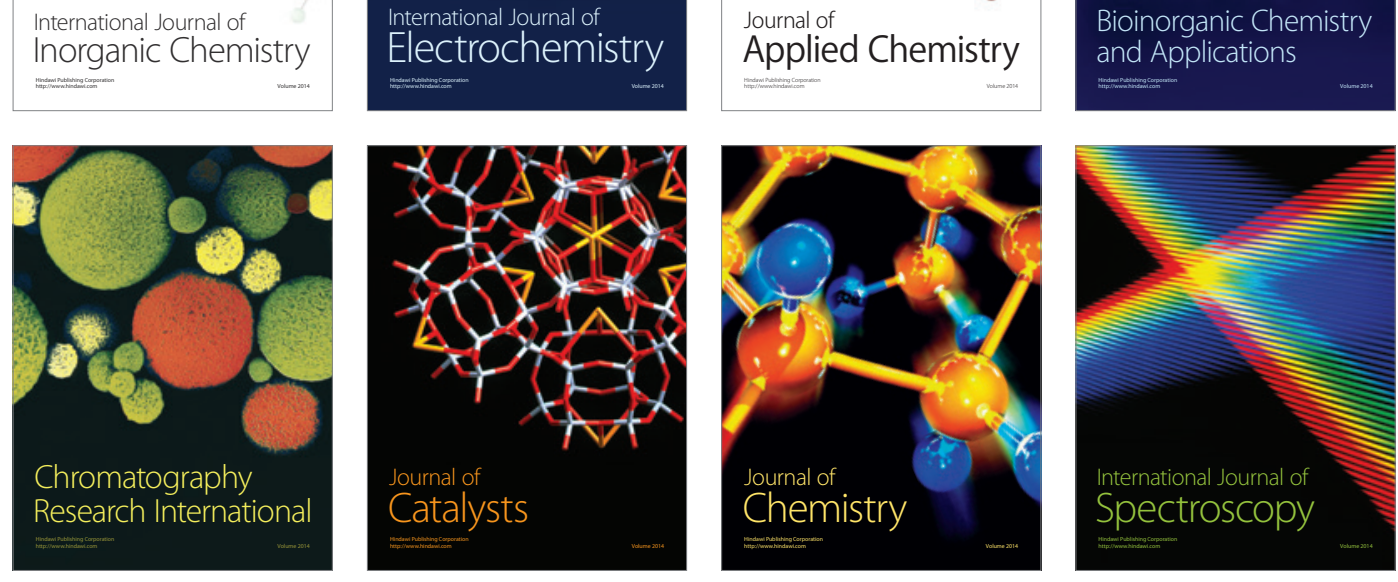